\title{
Hippocampal Activated Microglia May Contribute to Blood-Brain Barrier Impairment and Cognitive Dysfunction in Post-Traumatic Stress Disorder-Like Rats
}

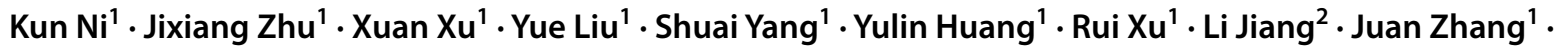 \\ Wei Zhang ${ }^{1} \cdot$ Zhengliang $\mathrm{Ma}^{1,2}$ (1)
}

Received: 11 November 2021 / Accepted: 2 February 2022 / Published online: 15 February 2022

(c) The Author(s), under exclusive licence to Springer Science+Business Media, LLC, part of Springer Nature 2022

\begin{abstract}
Post-traumatic stress disorder (PTSD)-associated cognitive dysfunction significantly disturbs patients' quality of life and will to live. However, its underlying mechanism is as yet unknown. Recent researches indicate that blood-brain barrier (BBB) breakdown is responsible for early cognitive dysfunction. Microglia might participate in remodeling of BBB-associated tight junction and regulating BBB integrity. Nevertheless, it is unclear whether microglia activation and BBB injury involve in PTSD-associated cognitive dysfunction. Hence, we established an animal model of PTSD, single prolonged stress (SPS), and investigated permeability changes in the hippocampus and further explored the effects of microglia on BBB remodeling. The Y maze was used to assess the changes of cognitive function. The sodium fluorescein (NaFlu) assay and western blotting analysis were employed to detect BBB integrity changes. Minocycline was administered to inhibit microglial activation. Immunofluorescence stains were used to assess the activation states in microglia. The results showed that SPS-exposed rats exhibited poorer cognitive performance, higher passage of $\mathrm{NaFlu}$, and lower expression of tight junction proteins (occludin and claudin 5) in the hippocampus on the day after SPS, but no difference on the 7th day. Inhibition of microglial activation by minocycline attenuated poor cognitive performance and BBB impairment including the extravasation of NaFlu and protein levels of the tight junction. Taken together, the present study indicates that BBB impairment may underlie the shared pathological basis of PTSD and cognitive dysfunction. Microglial activation may involve in BBB remodeling at the early stage of SPS.
\end{abstract}

Keywords Post-traumatic stress disorder $\cdot$ Single prolonged stress $\cdot$ Blood-brain barrier $\cdot$ Cognition dysfunction

Kun Ni and Jixiang Zhu should be regarded as joint first authors.

Juan Zhang

zhangjuannju@163.com

$\triangle$ Wei Zhang

genine@126.com

$\triangle$ Zhengliang Ma

mazhengliang1964@nju.edu.cn

1 Department of Anesthesiology, Drum Tower Hospital, Medical School of Nanjing University, 321 Zhongshan Road, Nanjing 210008, China

2 Nanjing Drum Tower Hospital Clinical College of Nanjing Medical University, 321 Zhongshan Road, Nanjing 210008, China

\section{Introduction}

Post-traumatic stress disorder (PTSD) is a complex and debilitating illness triggered by terrifying events, which is often companied by cognitive impairment, chronic pain, and affective disorders (Compean and Hamner 2019). The prevalence of PTSD in the general population is estimated at approximately $7 \%$ and higher among people experienced major traumatic events before (Zhou et al. 2021). For example, coronavirus disease 2019 (COVID-19) is a major source of traumatic stress for people around the globe since 2019. A meta-analysis revealed that the prevalence of PTSD among COVID-19 patients in the post-illness stage was up to $32.2 \%$ (Rogers et al. 2020). PTSD with negative alterations in cognition and mood was markedly associated with high morbidity and disability (Brown et al. 2020). Although cognitive dysfunction in PTSD patients has proved to be a 
non-negligible clinical problem, its underlying mechanism remains to be elucidated.

The blood-brain barrier (BBB) is the structural and functional interface that regulates substance and energy exchange in the central nervous system (CNS), thus maintaining the homeostasis of CNS (Villaseñor et al. 2019). It is mainly composed of brain microvascular endothelial cells, glial end-feet, pericytes, and the extracellular matrix that form the neurovascular unit with the adjacent neurons, astrocytes, and microglia (Iadecola 2017). Tight junctions between brain microvascular endothelial cells form the main anatomical basis of BBB and play an important role in limiting paracellular permeability (Rajagopal et al. 2019). Clinical studies showed that BBB breakdown in the hippocampus was a crucial characteristic of individuals with early cognitive dysfunction (Bowman et al. 2018; Nation et al. 2019; Montagne et al. 2020). Animal experiments also revealed that stress such as sleep deprivation and restraint stress could induce BBB hyper-permeability which was associated with emotional-cognitive disorders (Xue et al. 2019; Sántha et al. 2015; Xu et al. 2019). These researches prompt BBB injury might be a key mechanism of cognitive dysfunction.

Microglia is the rapid response cells coping with central nervous system injury including PTSD. Our previous study found that single prolonged stress (SPS), an animal model of PTSD, induced anxiety-like behavior and was accompanied by the activation of microglia in the CA1 and CA 3 regions of rat hippocampus, which could be reversed by prior administration of microglia inhibitor (minocycline) (Sun et al. 2016). Recent researches suggested microglia might contact with neuro-vasculature and crosstalk with endothelial to participate in remodeling of BBB-associated tight junction and regulating BBB integrity (Bisht et al. 2021; Delaney et al. 2021). Nevertheless, it is unclear whether microglia activation and BBB injury involve in PTSD-induced cognitive dysfunction. Therefore, we established SPS-induced cognitive dysfunction and investigated permeability changes in the hippocampus and further explored the effects of activated microglia on BBB remodeling.

\section{Materials and Methods}

\section{Experimental Animals}

Ninety adult male Sprague-Dawley rats (8 weeks, 210-230 g) were obtained from Charles River Animal Company (Zhejiang, China). Rats were housed in the barrier environment with free access to the diets and drinking water, light on between 8:00 and 20:00 h. All rats were allowed to acclimate to their environment for at least 1 week before the experimental procedure. Deep anesthesia was conducted with isoflurane before animals were sacrificed. Rats with abnormal behavioral features or dead rats during experiments should be excluded. All the experimental protocols were approved by the Ethics Committee of Drum Tower Hospital.

\section{Experimental Schemes}

All rats were randomly assigned to the following experiments as described (Fig. 1):

Experiment 1 Forty-two rats were randomized into two groups: group C (rats with no treatment) and group SPS (rats suffered from SPS). Nine rats were randomized picked to perform Y maze test on day 1 and day 7, respectively, after SPS treatment. After the behavior test, the brain samples were collected for western blot analysis $(n=6)$ and BBB permeability assessment $(n=3)$.
Fig. 1 The experimental designs for experiment 1 and experiment 2. Number of animals per group: Y-maze, $n=9$; NaFlu, $n=3$; WB, $n=6$; IF, $n=3$. NaFlu, sodium fluorescein assay; WB, western blotting; IF, immunofluorescence staining

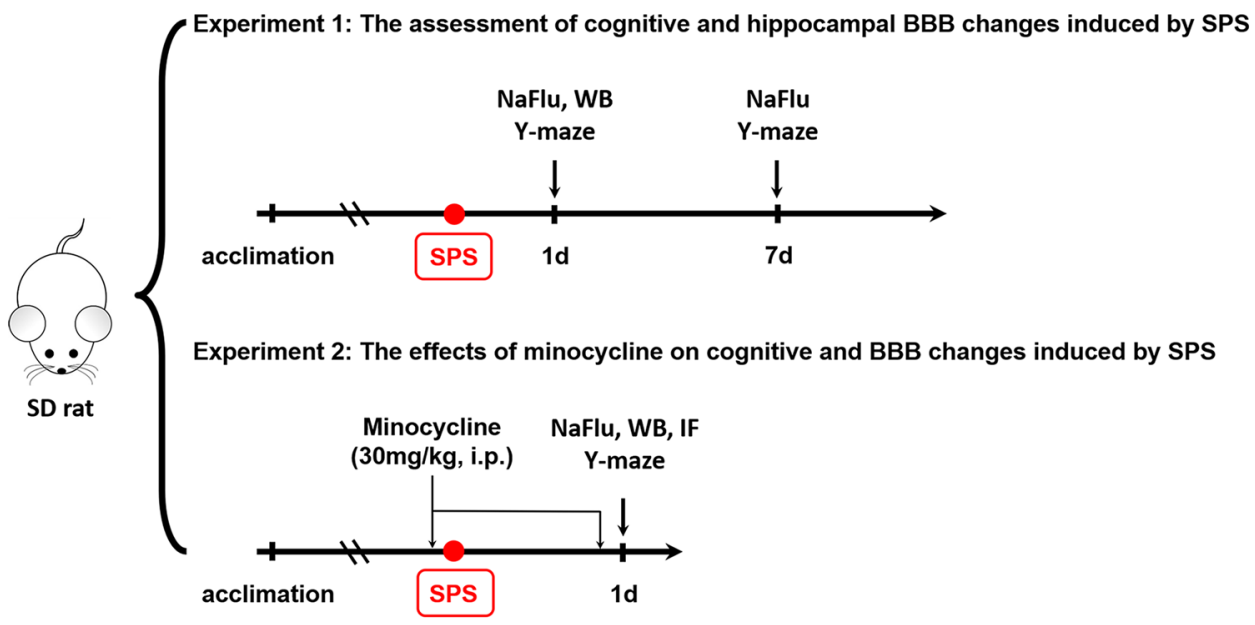


Experiment 2 Forty-eight SD rats were randomly assigned into 4 groups, group $\mathrm{C}+\mathrm{V}$ (control + vehicle, rats treated with saline), group $\mathrm{C}+\mathrm{M}$ (control + minocycline, rats treated with minocycline), group $\mathrm{S}+\mathrm{V}$ (SPS + vehicle, SPS-treated rats injected with saline), and group $\mathrm{S}+\mathrm{M}$ (SPS + minocycline, SPS-treated rats injected with minocycline). Rats received minocycline ( $30 \mathrm{mg} / \mathrm{kg}$ ) or an equal volume of saline intraperitoneally $30 \mathrm{~min}$ before and $23 \mathrm{~h}$ after SPS. Doses of systemic minocycline treatment were employed according to our previous study showing the potent inhibitory effect on activated microglia (Sun et al. 2016). The behavior test was conducted on the day after SPS $(n=9)$. After that, the brain samples were collected for immunofluorescence $(n=3)$, western blot analysis $(n=6)$, and BBB permeability assessment $(n=3)$.

\section{SPS Procedure}

The SPS procedure referred to a previous report (Sun et al. 2016). Rats were immobilized inside a plastic container for $2 \mathrm{~h}$ and then forced to swim in the clean water $\left(24{ }^{\circ} \mathrm{C}\right)$ for $20 \mathrm{~min}$. After swimming, the rats were dried and allowed a 15-min break. Finally, rats were anesthetized with ether until consciousness disappearance. Meanwhile, control rats were kept in the next room without any treatment.

\section{Y Maze Test}

Spontaneous alternation was used to assess the working memory in Y maze test (Li et al. 2019; Duchon et al. 2020). The Y maze consists of three arms $(50 \mathrm{~cm} \times 10 \mathrm{~cm} \times 30 \mathrm{~cm})$ at $120^{\circ}$ angles to each other. After habituating the behavior test room for $1 \mathrm{~h}$, rats were transferred to the center of $\mathrm{Y}$ maze to explore for 8 min freely. A video-tracking system was employed to record activity trajectories and the series of arm entries. Spontaneous alternation percentage was estimated by EthoVision XT software (Noldus Information Technology, Wageningen, Netherlands). Three consecutive entries into three different arms were defined as a success. Spontaneous alternation $(\%)=[$ (number of alternations) $/$ (total arm entries-2)] $\times 100 \%$.

\section{BBB Permeability Assessment}

BBB permeability was estimated by the sodium fluorescein (NaFlu) assay (Yang et al. 2014). Briefly, 10\% NaFlu (Sigma Aldrich, $2 \mathrm{ml} / \mathrm{kg}$ ) was injected via the tail vein and allowed to circulate for $30 \mathrm{~min}$. Subsequently, cold saline was perfused transcardially for $15 \mathrm{~min}$ to remove the intravascular dye after anesthesia. The hippocampus was sampled and weighed. Samples were homogenized in $500 \mu \mathrm{PBS}$ and vortexed thoroughly after adding equal volumes of $60 \%$ trichloroacetic. Samples, cooled for $30 \mathrm{~min}$, were centrifuged at $14,000 \times g$ for $10 \mathrm{~min}$ later. The concentration of $\mathrm{NaFlu}$ was detected (excitation wavelength, $440 \mathrm{~nm}$; emission wavelength, $525 \mathrm{~nm}$ ). A standard curve was drawn to link optical density and the concentration of NaFlu. BBB permeability was determined by the weight of $\mathrm{NaFlu}$ per gram of tissue.

\section{Immunofluorescence}

Rats were transcardially perfused with cold saline under deep anesthesia with isoflurane and then fixed with $10 \%$ buffered formalin phosphate. Whole brains were quickly removed and post-fixed overnight. Fixed samples were dehydrated in a gradient concentration of sucrose. Twentymicrometer frozen sections were balanced at room temperature for $15 \mathrm{~min}$ and washed in PBS for 5 min thrice. Sections were blocked with $10 \%$ goat serum for $1 \mathrm{~h}$ at room temperature. Subsequently, they were incubated with primary antibodies anti-Iba1 (1:500, Wako) overnight at $4{ }^{\circ} \mathrm{C}$ and were washed three times in PBS. Secondary antibodies, conjugated to AlexaFluor 488 were applied to slides to incubate for $1 \mathrm{~h}$ at room temperature. Stains were washed three times with PBS before nuclear staining with DAPI (Life Technologies). Microglial activation was reflected by comparing the numbers of Iba1-positive cells between groups. The numbers of Iba1-positive cell bodies, colocalized with DAPI, were counted manually (Sun et al. 2016).

\section{Western Blotting}

Western blotting was performed as described previously (Wang et al. 2020). Total protein from hippocampus was extracted by adding the ice-cold RIPA Lysis Buffer. Proteins were separated by gradient electrophoresis on 10-12.5\% SDS-PAGE gels and then transferred onto a polyvinylidene fluoride membrane (Millipore, USA). After they were blocked in 5\% skim milk, the membranes were incubated with the following primary antibodies overnight at $4{ }^{\circ} \mathrm{C}$ : claudin 5 (1:1000, Thermo Fisher, USA), occludin (1:10,000, Proteintech, China), Iba1 (1:500, Wako, Japan), and GAPDH (1:10,000, Proteintech, China). After washing with TBST, the corresponding secondary antibodies were applied for $1 \mathrm{~h}$ at room temperature (1:10,000, Millipore, USA). Membranes were visualized using ECL solution.

\section{Statistical Analysis}

The sample size was determined with PASS (power analysis and sample size, PASS) software (power $=0.8$, alpha $=0.05$ ). All parameters of the calculation and estimation of effect size were shown in supplement section (Table S1). Experimenters and investigators were blind to each group when 
Fig. 2 SPS-induced cognitive dysfunction. a Representative rats' movement traces were presented on days 1 and 7 after SPS between group $C$ and group SPS. b Spontaneous alternation was tested on days 1 and 7 after SPS between group $C$ and group SPS ( $n=9 /$ group). $* * P<0.01$, compared to group $\mathrm{C}$ a

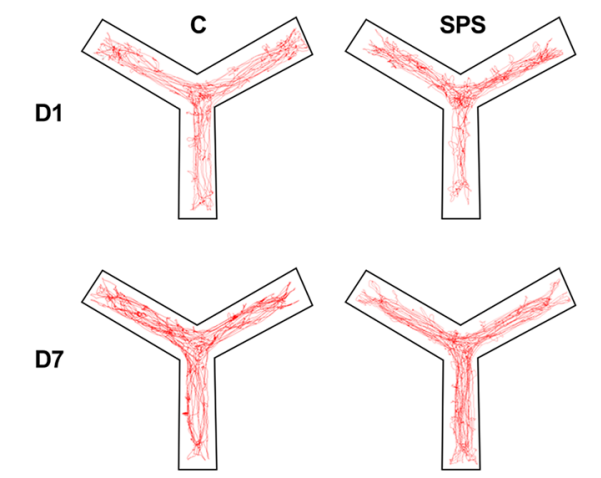

b

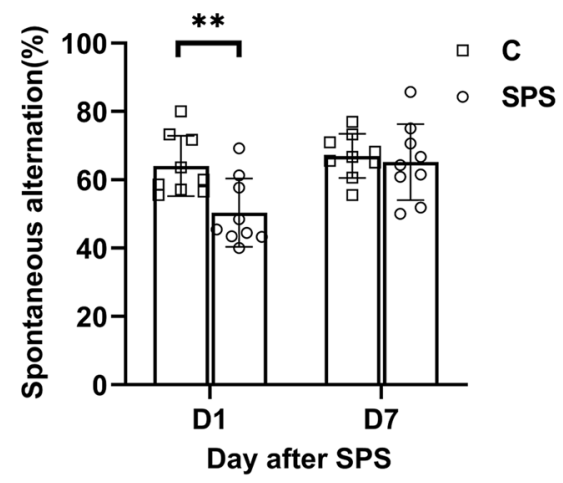

performing their respective procedures. Outlier testing was performed using Grubb's test by GraphPad Prism (Version 8.3.0). Data are presented as the means \pm SD. Nonparametric Student's t-test was used to compare the differences between groups. One-way ANOVA was performed to determine differences in res among groups. The statistical analysis was conducted using GraphPad Prism (Version 8.3.0). $P<0.05$ was considered statistically significant.

\section{Results}

\section{SPS-Induced Cognitive Impairment}

Spontaneous alternation test was performed in Y maze to assess the cognitive function. The typical movement trails of rats on days 1 and 7 after SPS are shown in Fig. 2a. Student's t-test revealed a significant difference in Y-maze spontaneous alternation of group SPS and group $\mathrm{C}$ on the day after SPS $(P<0.01$, Fig. 2b). However, there was no statistically significant difference between two groups at the 7 th day $(P>0.05$, Fig. 2b).

\section{SPS-Induced Hippocampal BBB Impairment}

To determine the changes of hippocampal BBB permeability, we detected the passage of NaFlu and levels of endothelial tight junction proteins of rats in group SPS and group C. NaFlu assay showed that the extravasation of NaFlu is higher in group SPS than in group $C$ on 1st day after SPS $(P<0.05)$, while no statistical difference was found on the 7 th day (Fig. 3a). Western blotting analysis revealed that the expression levels of claudin $5(P<0.01)$ and tight junction protein occludin $(P<0.001)$ significantly decreased in group SPS (Fig. 3b).

\section{Inhibition of Microglial Activation Attenuated Cognitive Impairment}

To investigate the effect of hippocampal activation of microglia in the pathogenesis of PTSD-associated cognitive dysfunction, minocycline was employed as its potential inhibition of microglial activation in the following experiment. Minocycline hydrochloride $(30 \mathrm{mg} / \mathrm{kg}$, i.p.) or the same volume of vehicle was injected at $30 \mathrm{~min}$ before and on the day after SPS to control and SPS-exposed rats. Spontaneous a

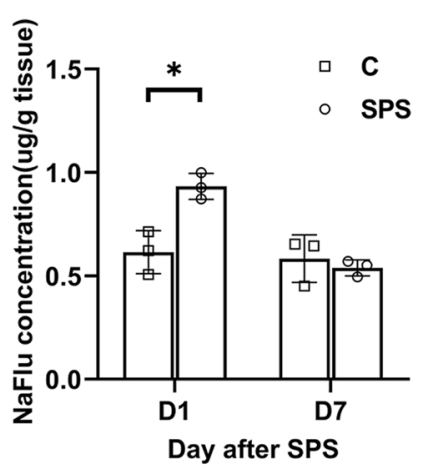

b
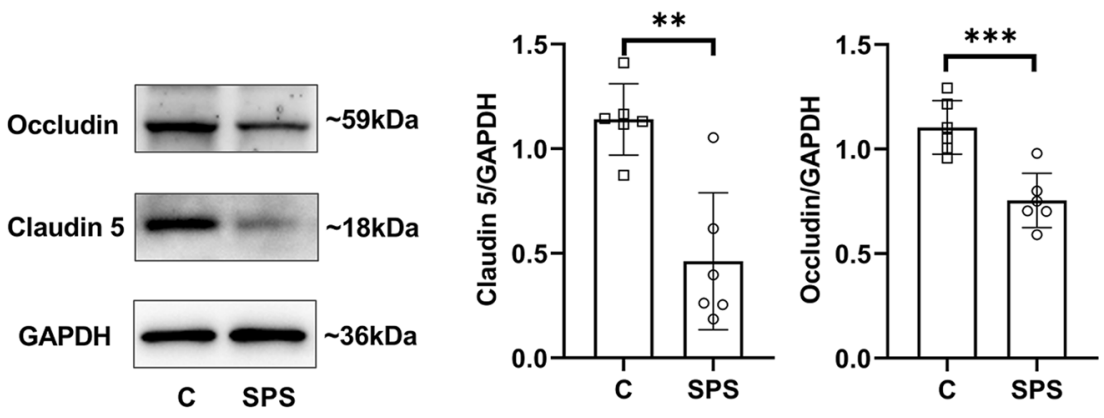

Fig. 3 SPS-induced hippocampal BBB impairment. a The NaFlu extravasation of hippocampus was tested on days 1 and 7 after SPS in each group ( $n=3$ /group). b Representative blots and quantification of tight junction proteins occludin and claudin 5 in the hippocampus on the first day after SPS ( $n=6 /$ group) $* P<0.05, * * P<0.01, * * * P<0.001$ 
alternation was measured on the day after SPS. The typical trajectory of each group rats on day 1 after SPS is shown in Fig. 4a. Compared to group $\mathrm{S}+\mathrm{V}$, minocycline improved the spontaneous alternation performance of rats exposed to SPS $(P<0.01$, Fig. 4 b). However, minocycline had no effect on cognitive behaviors in control rats $(P>0.05$, Fig. $4 \mathrm{~b})$.
Immunofluorescence was used to validate the morphological changes of microglia after SPS exposure. The number of positive stained cells of Ibal is calculated and summarized in Fig. 4c. Rats exposed to SPS presented an increased number of activated microglia in the hippocampus $(P<0.001$, Fig. $4 c)$, which were inhibited by minocycline $(P<0.001$,

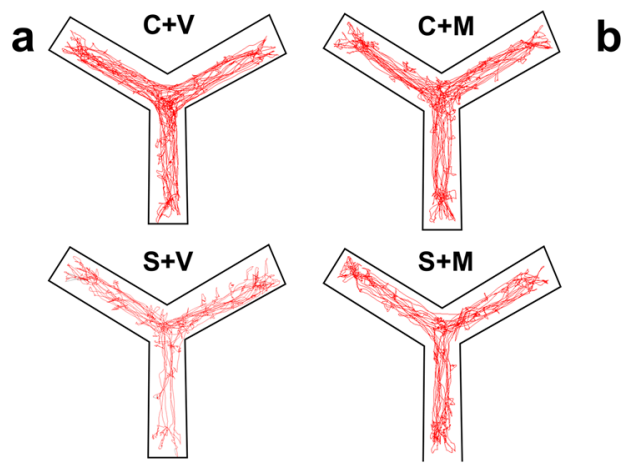

b

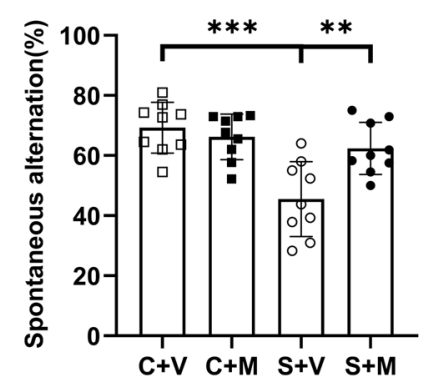

$\mathbf{C}+\mathrm{M}$

d
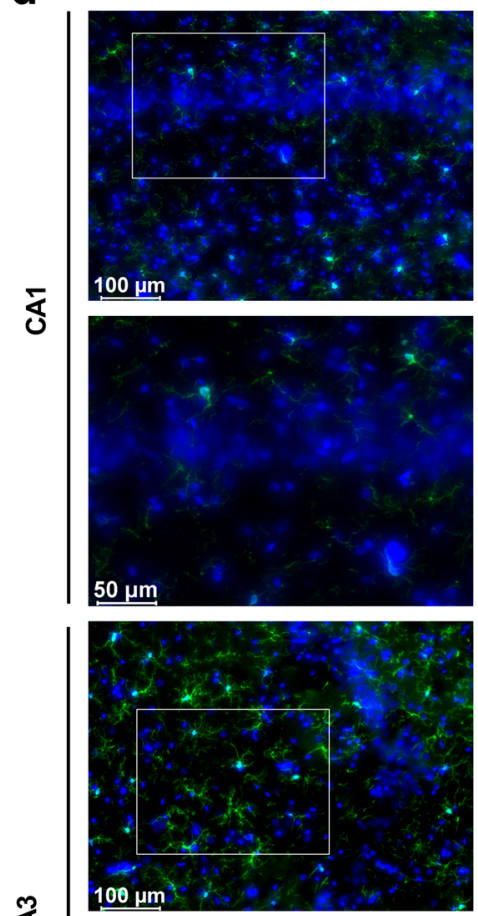

క̛⿱

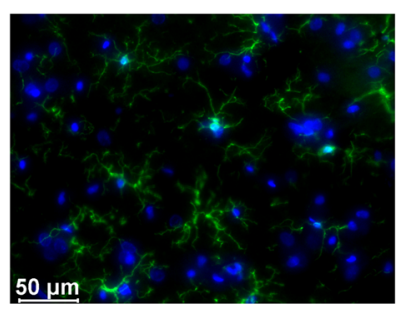

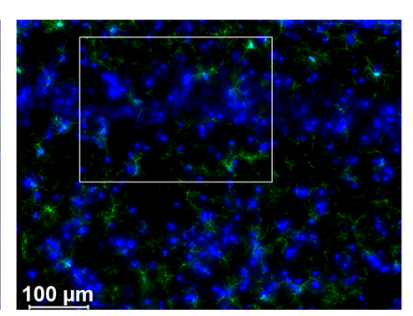
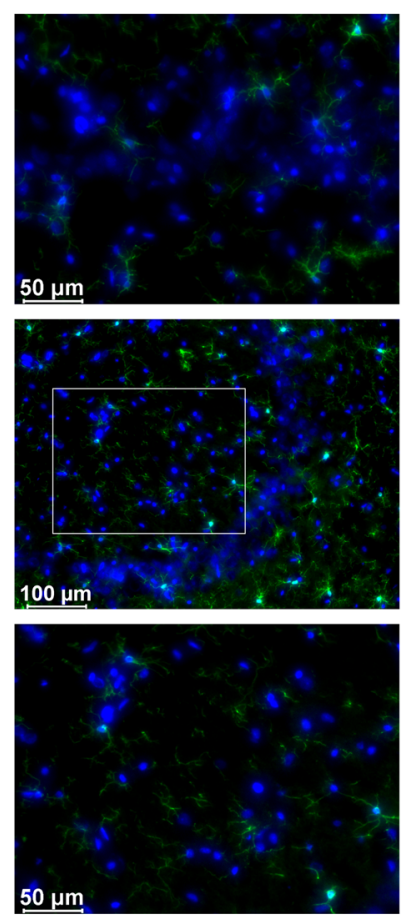

C

व $C+V \cdot C+M \circ S+V \cdot S+M$

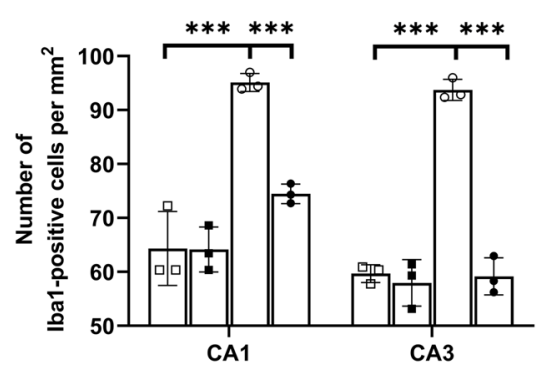

S+V
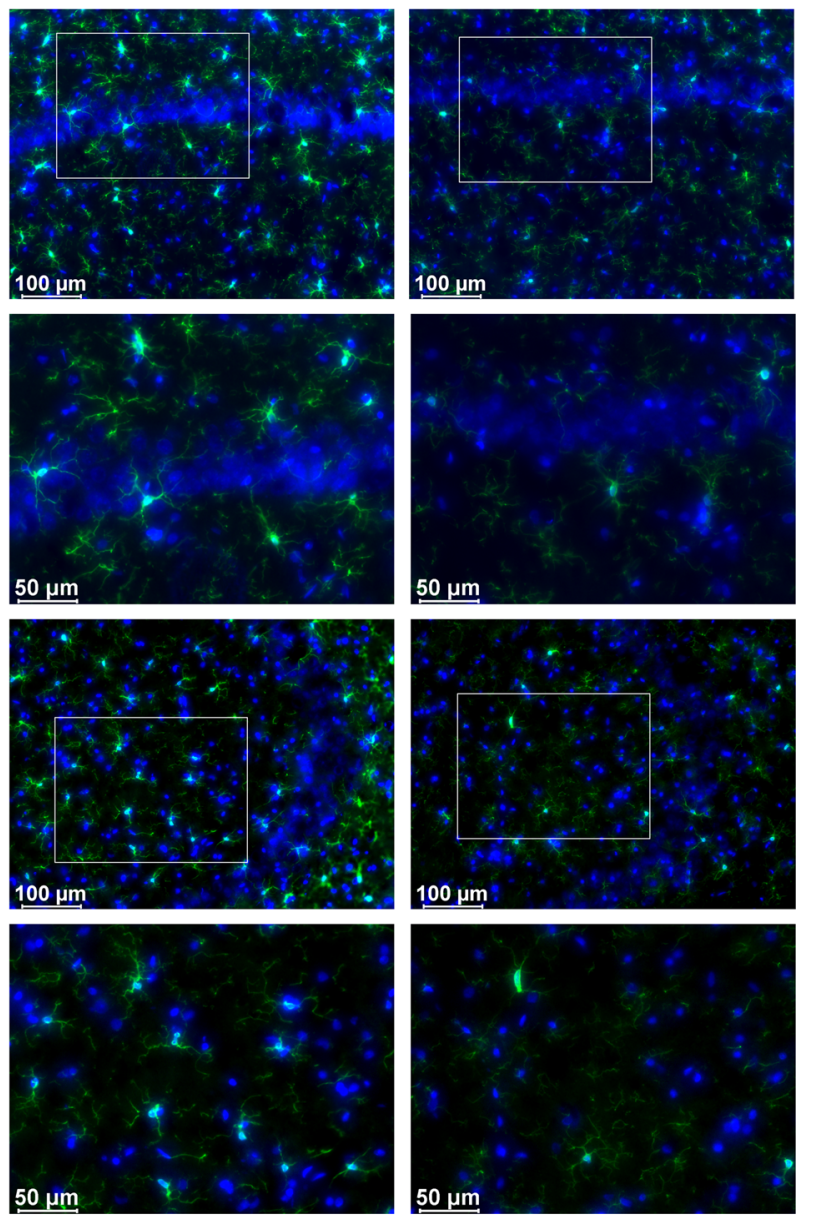

Fig. 4 Inhibition of microglia attenuated cognitive impairment. a Typical trajectories were presented on day 1 after SPS in control and SPS-exposed groups either treated with minocycline or vehicle intraperitoneally. b Spontaneous alternation was tested on day 1 after SPS in control and SPS-exposed groups either treated with minocycline or vehicle intraperitoneally ( $n=9 /$ group). $\mathbf{c}$ The number of Iba1-positive cell bodies was summarized (6 fields of 3 rats/group). d The representative Iba-1 (green) and DAPI (blue) stained images were captured in the CA1 and CA 3 regions of the hippocampus. ${ }^{*} P<0.05$, $* * P<0.01, * * * P<0.001$ 
a

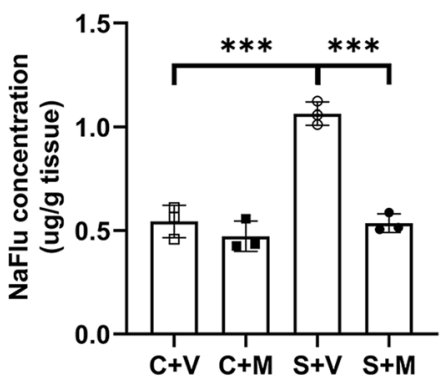

b

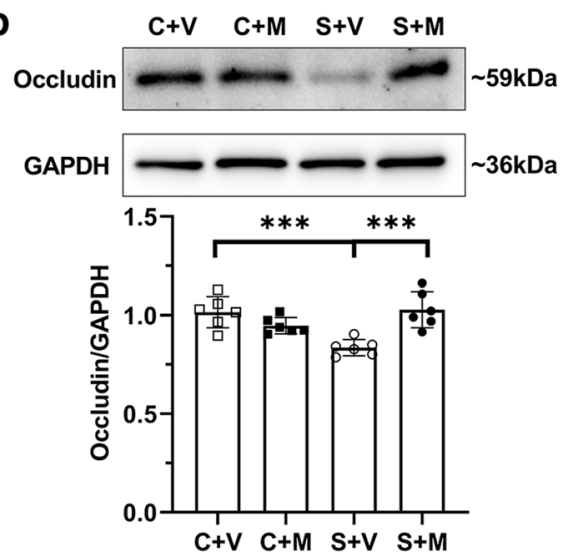

C

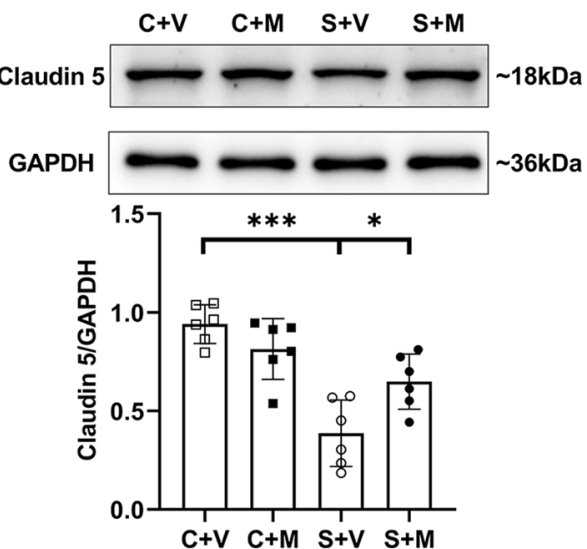

Fig. 5 Minocycline attenuated hippocampal BBB leakage. a The NaFlu extravasation in the hippocampus was tested on the day after SPS in control and SPS-exposed groups either treated with minocycline or vehicle intraperitoneally ( $n=3 /$ group). b Representative

Fig. 4c). The representative micrographs for Iba1 in the $\mathrm{CA} 1$ and $\mathrm{CA} 3$ regions of rat hippocampus in each group are shown in Fig. 4d.

\section{Inhibition of Microglial Activation Attenuated SPS-Induced BBB Injury}

To assess whether microglial activation involves in BBB breakdown, $\mathrm{NaFlu}$ assay and western blot analysis were conducted. Hippocampal content of fluorescein sodium reduced after being pretreated with minocycline in SPS-exposed groups (Fig. 5a). Compared with group $\mathrm{S}+\mathrm{V}$, decreased expression levels of occludin and claudin 5 were relieved by minocycline administration (Fig. 5b). Minocycline had no effect on BBB breakdown in control group both in NaFlu assay and western blot analysis $(P>0.05$, Fig. 5a, b).

\section{Discussion}

Our findings show that rats exhibit working memory impairment at the early stage after SPS. In the meantime, BBB integrity injury and microglial activation in the hippocampus were observed. Pretreated with minocycline, an inhibitor of activated microglia not only inhibited the activation of microglia but also improved the hippocampal BBB disruption. Likewise, poor working memory in SPS-treated rats was also attenuated by minocycline. Our results suggest that the activated microglia may contribute to SPS-induced BBB remodeling and working memory impairment.

Compared to the control group, the percentage of spontaneous alternation was significantly decreased on the first day after SPS. But no obvious difference was observed between groups on day 7 . The behavior results indicate blots and quantification of tight junction protein levels of occludin and claudin 5 in the hippocampus on the first day after SPS were presented ( $n=6 /$ group). $* P<0.05, * * P<0.01, * * * P<0.001$

that SPS may induce working memory impairment at the early stage, which is consistent with other research (Han et al. 2015). Meanwhile, the content of NaFlu and the protein levels of occludin and claudin 5 in the hippocampus showed markedly increasing and decreasing respectively in SPS group. These suggest that the integrity of hippocampal BBB was impaired in SPS condition. As another animal model of strong psychological stress, restraint stress-treated rats also performed decreased expression and discontinuity of the occludin and claudin 5 in the hippocampus (Sántha et al. 2015). A network-level analysis of the hippocampus in the social defeat model for evaluating PTSD symptoms showed notable damage in tight junctions as well (Yang et al. 2013). Clinical observations provided analogous clues that the increased level of S-100 $\beta$ (a biomarker of BBB disruption) in the serum was associated with PTSD-like symptoms (Sojka et al. 2006). The above results indicate together that BBB impairment might be the crucial point of the generation of PTSD-related behaviors. However, a recent study presented results inconsistent with ours that the CSF/serum albumin ratio of SPS-treated rats showed no distinct differences with control group (Dib et al. 2021). This may be attributed to the size of tracer molecules that albumin has a larger molecular weight than NaFlu (66,458 Da vs. $376 \mathrm{Da})$.

Minocycline, a specific inhibitor of microglial activation, has been shown to present neuroprotective effects in various models of neurodegenerative diseases. Our previous study revealed that minocycline suppressed SPS-induced microglia activation in the hippocampus and attenuated both SPSinduced pain and anxiety-like behaviors (Sun et al. 2016). In this experiment, intraperitoneal injection of minocycline not only mitigated working memory impairment and improved the hippocampal BBB damage in SPS condition. Consistent with our studies, minocycline also attenuated hippocampal 
microglia activation and PTSD-related behavioral changes including cognition, anxiety, and pain-related parameters in other studies (Sun et al. 2016; Wang et al. 2018; Rooney et al. 2020). Several researches have documented that microgliaendothelial crosstalk is necessary for the regulation of BBB function. Wang et al. reported that minocycline might preserve BBB via decreasing DKK1 expression in microglia involving Wnt $1-\beta$-catenin signaling (Wang et al. 2019). Wnt1- $\beta$-catenin pathway plays a crucial part in regulating the composition of endothelial tight junctions such as claudin 5, occludin, and ZO-1 (Chang et al. 2017; Luo et al. 2020). Koichiro et al. reported that microglia enhanced BBB integrity via expression of claudin 5 in the early inflammatory response and impaired BBB function by phagocytosing astrocytic end-feet during the sustained inflammation stage (Haruwaka et al. 2019). The findings suggest microglia plays a dual role in the regulation of BBB integrity. These indicate that activated microglia, at least in part, are involved in stress-induced BBB remodeling.

Of course, there are some limitations to the study. Firstly, considering the potential hippocampal injury through local minocycline delivery, systemic administration was used in the study. Although this way is lack of tissue specificity, it avoids the impact on cognitive performance and BBB permeability detection. Secondly, though minocycline is a valid microglial inhibitor, some studies indicated that minocycline can also act on astrocytes and possess systematic antiinflammation effects (Keller et al. 2011; Lavin Plaza et al. 2020). Therefore, we will take more tissue- and cell-specific strategies to further confirm the current results.

In summary, the present study indicates that BBB impairment may be the link between PTSD and cognitive dysfunction. Microglial activation may be responsible for BBB remodeling at the early stage of SPS. We hope our findings can bring a new perspective to the research field of PTSDrelated cognitive dysfunction.

Supplementary Information The online version contains supplementary material available at https://doi.org/10.1007/s12031-022-01981-4.

Acknowledgements All authors listed are grateful for the technical and instrument support provided by the Department of Scientific Research, Drum Tower Hospital. We also thank Dr. Liangyu Peng and Rui Dong for their kind suggestions about the analysis of immunofluorescence images.

Author Contribution Study concept and design: Zhengliang Ma, Wei Zhang, and Juan Zhang. Analysis and interpretation of data and drafting of manuscript: Kun Ni and Jixiang Zhu. Experiment implementation: Kun Ni, Jixiang Zhu, Xuan Xu, Yue Liu, Shuai Yang, Yulin Huang, Rui Xu, and Li Jiang.

Funding This work was supported by the China National Natural Science Foundation (81971044, 82171225, 81771142, 81870875).

Availability of Data and Materials The data is provided upon request.

\section{Declarations}

Ethics Approval and Consent to Participate Animal experiments were performed according to protocols approved by the Ethics Committee of Drum Tower Hospital, and all efforts were made to minimize the number of animals used and their suffering.

Consent for Publication All authors listed agree to publish this article.

Competing Interests The authors declare no competing interests.

\section{References}

Bisht K, Okojie KA, Sharma K et al (2021) Capillary-associated microglia regulate vascular structure and function through PANX1-P2RY12 coupling in mice. Nat Commun 12:5289. https://doi.org/10.1038/ s41467-021-25590-8

Brown LA, Chen S, Narine K et al (2020) DSM-5 PTSD symptom clusters and suicidal ideation in veterans. Psychiatry Res 288:112942. https://doi.org/10.1016/j.psychres.2020.112942

Bowman GL, Dayon L, Kirkland R et al (2018) Blood-brain barrier breakdown, neuroinflammation, and cognitive decline in older adults. Alzheimers Dement 14:1640-1650. https://doi.org/10. 1016/j.jalz.2018.06.2857

Chang J, Mancuso MR, Maier C et al (2017) Gpr124 is essential for blood-brain barrier integrity in central nervous system disease. Nat Med 23:450-460. https://doi.org/10.1038/nm.4309

Compean E, Hamner M, (2019) Posttraumatic stress disorder with secondary psychotic features (PTSD-SP): diagnostic and treatment challenges. Prog Neuropsychopharmacol Biol Psychiatry 88:265-275. https://doi.org/10.1016/j.pnpbp.2018.08.001

Delaney C, Farrell M, Doherty CP et al (2021) Attenuated CSF-1R signalling drives cerebrovascular pathology. EMBO Mol Med 13:e12889. https://doi.org/10.15252/emmm.202012889

Dib P, Zhang Y, Ihnat MA et al (2021) TNF-alpha as an initiator of allodynia and anxiety-like behaviors in a preclinical model of PTSD and comorbid pain. Front Psychiatry 12:721999. https:// doi.org/10.3389/fpsyt.2021.721999

Duchon A, Gruart A, Albac C et al (2020) Long-lasting correction of in vivo LTP and cognitive deficits of mice modelling Down syndrome with an $\alpha 5$-selective GABA(A) inverse agonist. Br J Pharmacol 177:1106-1118. https://doi.org/10.1111/bph.14903

Han F, Xiao B, Wen L (2015) Loss of glial cells of the hippocampus in a rat model of post-traumatic stress disorder. Neurochem Res 40:942-951. https://doi.org/10.1007/s11064-015-1549-6

Haruwaka K, Ikegami A, Tachibana Y et al (2019) Dual microglia effects on blood brain barrier permeability induced by systemic inflammation. Nat Commun 10:5816. https://doi.org/10.1038/ s41467-019-13812-z

Iadecola C (2017) The neurovascular unit coming of age: a journey through neurovascular coupling in health and disease. Neuron 96:17-42. https://doi.org/10.1016/j.neuron.2017.07.030

Keller AF, Gravel M, Kriz J (2011) Treatment with minocycline after disease onset alters astrocyte reactivity and increases microgliosis in SOD1 mutant mice. Exp Neurol 228:69-79. https://doi.org/10. 1016/j.expneurol.2010.12.010

Lavin Plaza B, Phinikaridou A, Andia ME et al (2020) Sustained focal vascular inflammation accelerates atherosclerosis in remote arteries. Arterioscler Thromb Vasc Biol 40:2159-2170. https://doi.org/ 10.1161/atvbaha.120.314387

Li J, Liu Y, Liu B et al (2019) Mechanisms of aerobic exercise upregulating the expression of hippocampal synaptic plasticity-associated 
proteins in diabetic rats. Neural Plast 2019:7920540. https://doi. org/10.1155/2019/7920540

Luo X, Li L, Zheng W et al (2020) HLY78 protects blood-brain barrier integrity through $\mathrm{Wnt} / \beta$-catenin signaling pathway following subarachnoid hemorrhage in rats. Brain Res Bull 162:107-114. https://doi.org/10.1016/j.brainresbull.2020.06.003

Montagne A, Nation DA, Sagare AP et al (2020) APOE4 leads to blood-brain barrier dysfunction predicting cognitive decline. Nature 581:71-76. https://doi.org/10.1038/s41586-020-2247-3

Nation DA, Sweeney MD, Montagne A et al (2019) Blood-brain barrier breakdown is an early biomarker of human cognitive dysfunction. Nat Med 25:270-276. https://doi.org/10.1038/s41591-018-0297-y

Rajagopal N, Irudayanathan FJ, Nangia S (2019) Computational nanoscopy of tight junctions at the blood-brain barrier interface. Int $\mathbf{J}$ Mol Sci 20. https://doi.org/10.3390/ijms20225583

Rogers JP, Chesney E, Oliver D et al (2020) Psychiatric and neuropsychiatric presentations associated with severe coronavirus infections: a systematic review and meta-analysis with comparison to the COVID-19 pandemic. Lancet Psychiatry 7:611-627. https:// doi.org/10.1016/s2215-0366(20)30203-0

Rooney S, Sah A, Unger MS et al (2020) Neuroinflammatory alterations in trait anxiety: modulatory effects of minocycline. Transl Psychiatry 10:256. https://doi.org/10.1038/s41398-020-00942-y

Sántha P, Veszelka S, Hoyk Z et al (2015) Restraint stress-induced morphological changes at the blood-brain barrier in adult rats. Front Mol Neurosci 8:88. https://doi.org/10.3389/fnmol.2015. 00088

Sojka P, STåLNACKE B-M, BJöRNSTIG U, et al (2006) One-year follow-up of patients with mild traumatic brain injury: occurrence of post-traumatic stress-related symptoms at follow-up and serum levels of cortisol, S-100B and neuron-specific enolase in acute phase. Brain Inj 20:613-620

Sun R, Zhang Z, Lei Y et al (2016) Hippocampal activation of microglia may underlie the shared neurobiology of comorbid posttraumatic stress disorder and chronic pain. Mol Pain 12. https://doi. org/10.1177/1744806916679166

Villaseñor R, Lampe J, Schwaninger M et al (2019) Intracellular transport and regulation of transcytosis across the blood-brain barrier. Cell Mol Life Sci 76:1081-1092. https://doi.org/10.1007/ s00018-018-2982-x

Wang G, Li Z, Li S et al (2019) Minocycline preserves the integrity and permeability of BBB by altering the activity of DKK1-Wnt signaling in ICH model. Neuroscience 415:135-146. https://doi. org/10.1016/j.neuroscience.2019.06.038

Wang W, Wang R, Xu J et al (2018) Minocycline attenuates stressinduced behavioral changes via its anti-inflammatory effects in an animal model of post-traumatic stress disorder. Front Psych 9. https://doi.org/10.3389/fpsyt.2018.00558

Wang C, Xu K, Wang Y et al (2020) Spinal cannabinoid receptor 2 activation reduces hypersensitivity associated with bone cancer pain and improves the integrity of the blood-spinal cord barrier. Reg Anesth Pain Med 45:783-791. https://doi.org/10.1136/ rapm-2019-101262

Xu G, Li Y, Ma C et al (2019) Restraint stress induced hyperpermeability and damage of the blood-brain barrier in the amygdala of adult rats. Front Mol Neurosci 12:32. https://doi.org/10.3389/ fnmol.2019.00032

Xue R, Wan Y, Sun X et al (2019) Nicotinic mitigation of neuroinflammation and oxidative stress after chronic sleep deprivation. Front Immunol 10:2546. https://doi.org/10.3389/fimmu.2019.02546

Yang R, Daigle BJ, Muhie SY Jr et al (2013) Core modular blood and brain biomarkers in social defeat mouse model for post traumatic stress disorder. BMC Syst Biol 7:80. https://doi.org/10.1186/ 1752-0509-7-80

Yang M, Rainone A, Shi XQ et al (2014) A new animal model of spontaneous autoimmune peripheral polyneuropathy: implications for Guillain-Barré syndrome. Acta Neuropathol Commun 2:5-5. https://doi.org/10.1186/2051-5960-2-5

Zhou Y-G, Shang Z-L, Zhang F et al (2021) PTSD: past, present and future implications for China. Chin J Traumatol 24:187-208. https://doi.org/10.1016/j.cjtee.2021.04.011

Publisher's Note Springer Nature remains neutral with regard to jurisdictional claims in published maps and institutional affiliations. 